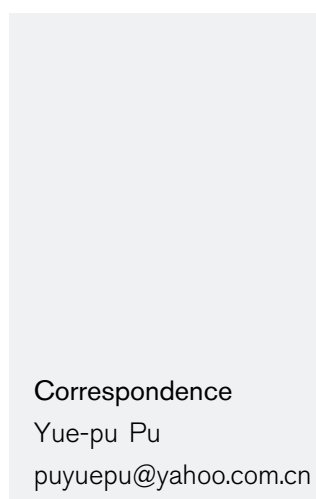

Received 5 November 2005 Accepted 1 June 2006

\section{Antimicrobial susceptibility of Neisseria gonorrhoeae isolated in Jiangsu Province, China, with a focus on fluoroquinolone resistance}

\author{
Bei Wang, ${ }^{1}$ Jin-shui $\mathrm{Xu},{ }^{1}$ Chang-xian Wang, ${ }^{1}$ Zu-huang $\mathrm{Mi}^{2}{ }^{2}$ Yue-pu $\mathrm{Pu},{ }^{1}$ \\ Mamie Hui, ${ }^{3}$ Thomas K. W. Ling ${ }^{3}$ and Chiu-Yeung Chan ${ }^{3}$ \\ ${ }^{1}$ School of Public Health, Southeast University, Nanjing, Jiangsu, People's Republic of China \\ ${ }^{2}$ Wuxi Clon-Gen Technique Institute, Wuxi, Jiangsu, People's Republic of China \\ ${ }^{3}$ Department of Microbiology, The Chinese University of Hong Kong, Prince of Wales Hospital, \\ Hong Kong Special Adminstrative Region, People's Republic of China
}

\begin{abstract}
In this study, the phenotypic and genotypic resistance to fluoroquinolones in Neisseria gonorrhoeae isolated in Jiangsu Province, China, was analysed. In vitro susceptibility testing of eight antimicrobial agents, including ciprofloxacin and levofloxacin, against 95 clinical isolates was carried out. Detection of mutations in the $g y r A$ and parC genes was performed by sequence analysis. The clinical isolates demonstrated $100 \%$ resistance to ciprofloxacin and $98.9 \%$ non-susceptibility to levofloxacin. All of the isolates were susceptible to cefotaxime and ceftriaxone. For cefepime, spectinomycin and tetracycline, 98.9, 94.7 and $1 \cdot 1 \%$ of the isolates were susceptible, respectively. None of the isolates was susceptible to penicillin. Five types based on gyrA mutations could be categorized among 54 isolates with seven different mutation sites found on their parC gene. Analysis of sequence results showed that the gyrA mutation Asp-95 $\rightarrow$ Ala and the parC mutations Ser-87 $\rightarrow$ Arg and Ser-87 $\rightarrow$ Asn made a significant contribution to the resistance to fluoroquinolones, in addition to double mutations found in each gene. Therefore, the use of fluoroquinolones in the treatment of $N$. gonorrhoeae infections in Jiangsu Province is not recommended, while the use of third- and fourth-generation cephalosporins and spectinomycin is recommended.
\end{abstract}

\section{INTRODUCTION}

Neisseria gonorrhoeae is a major pathogen of sexually transmitted diseases in China, with a cumulative total of 185319 notified cases in 2003 (Chinese Centre for Disease Control and Prevention, 2004). Nowadays, treatment of gonorrhoea using chloramphenicol, sulphonamide, penicillin and tetracycline has become ineffective. The fluoroquinolones were introduced and widely used clinically in the 1980s because of their effectiveness and convenience in single-dose oral administration, together with lower cost. After 10 years of clinical application, an increasing trend of fluoroquinolone resistance in N. gonorrhoeae has been reported in Japan, Korea, south-east Asia, Canada and China (Krishna et al., 2005; Ng et al., 2002; Tanaka et al., 2004; World Health Organization, 2003; Yoo et al., 2004). In China, the rate of fluoroquinolone resistance is about 40-80 \%, depending on the region studied ( $\mathrm{Gu}$ et al., 2004; Ye, 2001). However, in China, quinolones are still recommended to treat gonorrhoea (single dose $500 \mathrm{mg}$ for ciprofloxacin) (Cai et al., 2003; Su, 2002; Su et al., 2004; World Health Organization, 2003; Zhu \& Zhao, 2003). Accordingly, it is necessary to investigate the resistance rate and mechanism in $N$. gonorrhoeae. It has been reported that the development of mutations in DNA gyrase subunit A (GyrA) encoded by the gyrA gene and mutations in topoisomerase IV encoded by the parC gene account for most ciprofloxacin resistance (Belland et al., 1994). We collected 95 isolates of $N$. gonorrhoeae from three regions of Jiangsu Province during 2003 to study their susceptibilities to eight antimicrobial agents and assess the correlation between fluoroquinolone MICs and amino acid changes in GyrA and ParC.

\section{METHODS}

Bacterial isolates and culture. Ninety-five non-duplicate isolates of $N$. gonorrhoeae were collected from patients who attended sexually transmitted disease (STD) clinics in the Nanjing, Xuzhou and Wuxi regions between May and December 2003. The organisms were cultured and isolated on NG-Thayer-Martin agar (bioMérieux), and identified by Gram stain and oxidase reaction, followed by carbohydrate-utilization studies of glucose, maltose, fructose and sucrose (API NH, bioMérieux). The bacterial isolates were kept on chocolate agar slants (bioMérieux) for short-term storage. Pure cultures supplemented with $8 \%$ skimmed milk and $10 \%$ fetal calf serum were used for long-term storage at $-70{ }^{\circ} \mathrm{C}$. 
In vitro antimicrobial susceptibility testing. Material of known potency of the following antimicrobial agents was used for MIC determination: ciprofloxacin (Bayer), levofloxacin (Daiichi), spectinomycin (Pharmacia and Upjohn), penicillin (Sigma), tetracycline (Sigma), cefotaxime (Sigma), cefepime (Bristol-Myers-Squibb) and ceftriaxone (Sigma). MIC values were determined by an agar dilution method with GC agar base (Oxoid) plus supplements according to the National Committee for Clinical Laboratory Standards (2002). A multipoint inoculator (MIC-2000 System, Dynatech) was used to deliver $10^{4}$ c.f.u. per spot onto GC agar base supplemented with $1 \%$ Vitox (Oxoid) and $5 \%$ lysed horse blood. N. gonorrhoeae ATCC 49226 was included as the control strain for the antimicrobial susceptibility test. The MIC $\left(\mathrm{mg} \mathrm{l}^{-1}\right)$ was defined as the concentration at which no colony was detected after agar plates were incubated at $37^{\circ} \mathrm{C}$ in $5 \% \mathrm{CO}_{2}$ for $24 \mathrm{~h}$. Resistance breakpoints for the antimicrobial agents penicillin, cefotaxime, ceftriaxone, cefepime, tetracycline, ciprofloxacin and spectinomycin were interpreted according to the National Committee for Clinical Laboratory Standards (2002). For levofloxacin, MIC values of $\geqslant 1 \mathrm{mg} \mathrm{l}^{-1}$ were considered to be resistant (Shigemura et al., 2004; Centers for Disease Control and Prevention, 2005).

Detection of mutations in gyrA and parC genes. Bacterial isolates were selected from every ciprofloxacin MIC level by the use of a stratified random-sampling method. Bacterial cells were harvested and suspended in Tris/EDTA buffer. Cell suspension $(100 \mu \mathrm{l})$ was centrifuged at $10000 \mathrm{~g}$ at $4{ }^{\circ} \mathrm{C}$ for $5 \mathrm{~min}$, the pellet was resuspended in lysing buffer (Tris/EDTA containing $200 \mathrm{ng}$ proteinase $\mathrm{K} \mathrm{ml}^{-1}$ ) at $55^{\circ} \mathrm{C}$ for $1 \mathrm{~h}$, and then incubated at $95^{\circ} \mathrm{C}$ for $5 \mathrm{~min}$, centrifuged at $10000 \mathrm{~g}$ for $5 \mathrm{~min}$, and the supernatant collected and used as template. Primers for the gyrA gene were: forward $5^{\prime}$-GTA CTG TAC GCG ATG CAC GA-3' and reverse 5'-CGA GCC GTT GAC GAG CAG T- $3^{\prime}$, leading to a product of $372 \mathrm{bp}$. The amplification kit was obtained from the Wuxi Clon-Gen Technique Institute with the following protocol: $94{ }^{\circ} \mathrm{C}$ denaturation for $2 \mathrm{~min}$, followed by 35 cycles of denaturation at $94^{\circ} \mathrm{C}$ for $30 \mathrm{~s}$, annealing at $55^{\circ} \mathrm{C}$ for $30 \mathrm{~s}$ and extension at $72{ }^{\circ} \mathrm{C}$ for $60 \mathrm{~s}$, with final extension at $72{ }^{\circ} \mathrm{C}$ for 2 min. Primers for amplification of the parC gene were: forward $5^{\prime}$ GCA CGC TTC CCA TAC CGA-3' and reverse 5'-TCC ACC GTC CCC TGA TTG-3', leading to a product of $410 \mathrm{bp}$. The amplification protocol for the parC gene was identical to that for the gyrA gene, with the exception of denaturation at $93^{\circ} \mathrm{C}$. The primers for gyrA and parC were designed according to primer design principles and compared with sequences of homologous genes in GenBank. The amplified fragment of gyrA is from position 175-546, that of parC from position 15-443. The PCR products were sent to Shanghai BioAsia Biotechnology for sequence analysis on an ABI377 automatic sequencer (Applied Biosystems). The PCR product sequences were aligned, analysed and compared with the sequence of a wild-type strain of $N$. gonorrhoeae (GenBank accession nos U08817 and U08907) by Bioedit software (Ibis Therapeutics).

Data analysis. SAS 8.1 software (SAS Institute) was used for ANOVA analysis and multiple regression analysis of correlation between mutation sites and MIC results.

\section{RESULTS AND DISCUSSION}

The 95 non-duplicate $N$. gonorrhoeae isolates were collected from 78 males and 17 females, with a mean age of 33.96 years (SD $10 \cdot 49$ years).

The antimicrobial MIC distribution for 95 isolates of N. gonorrhoeae is shown in Table 1. All of the isolates were susceptible to cefotaxime and ceftriaxone. One isolate was susceptible to tetracycline. None of the isolates was

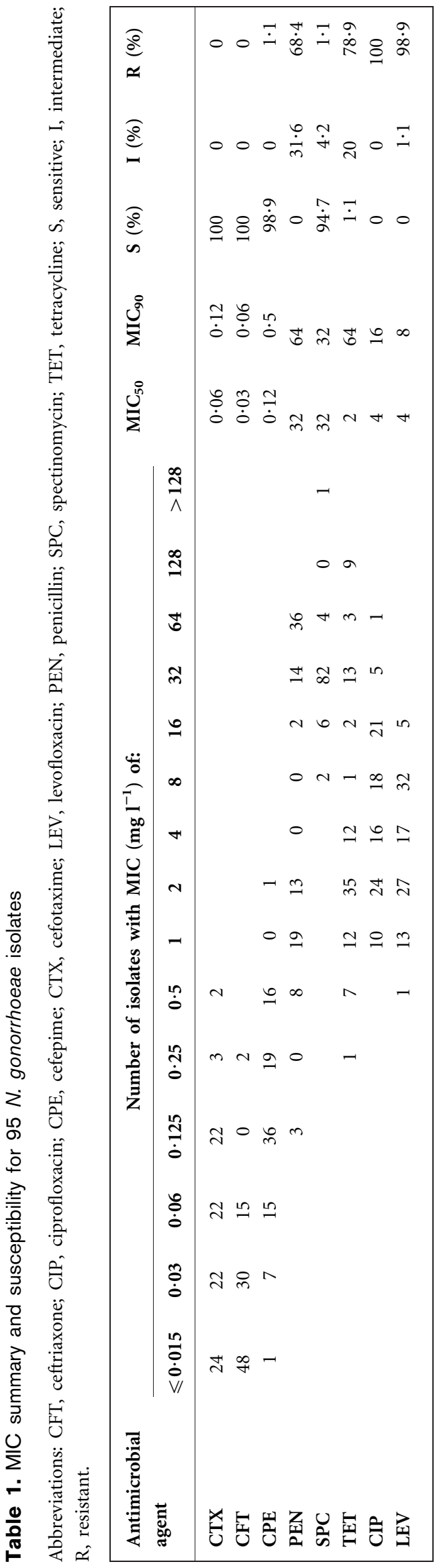


susceptible to penicillin. Spectinomycin resistance was very low: only one isolate showed an MIC $>128 \mathrm{mg} \mathrm{l}^{-1}$. One isolate was resistant to cefepime with $\mathrm{MIC}=2 \mathrm{mg} \mathrm{l}^{-1}$. All isolates were considered resistant to ciprofloxacin and non-susceptible to levofloxacin (Table 1).

Of the 95 isolates, 60 were selected according to the ciprofloxacin MIC distribution. However, two of these could not be amplified and four could not be sequenced. As a result, 54 isolates were selected for PCR of amino acid changes in the GyrA and ParC subunits. Five types of gyrA mutations could be categorized, with amino acid changes mainly found in positions 91 and 95 . Eleven mutation sites were found on the parC gene, leading to seven amino acid changes (Table 2), Four silent mutations were also detected (bases $312 \mathrm{~T} \rightarrow \mathrm{C}$, $387 \mathrm{G} \rightarrow \mathrm{A}, 393 \mathrm{C} \rightarrow \mathrm{G}$ and $414 \mathrm{G} \rightarrow \mathrm{A}$ ) that gave no amino acid change.

Statistical analysis of the relationships between different mutations in the gyrA and parC genes, and levels of fluoroquinolone resistance, revealed significant correlations. It was noted that when more mutations were found in the two genes of a particular isolate, the organism became more resistant, with a higher MIC, as found with other bacterial species (Hawkey, 2003). Isolates with only gyrA mutations had lower MICs than those with mutations in both gyrA and $\operatorname{par} C(P<0.05$ for ciprofloxacin; Table 3$)$. In addition, analysis also revealed that mutations in gyrA resulting in Asp-95 $\rightarrow$ Ala and in parC resulting in Ser-87 $\rightarrow$ Arg and Ser-87 $\rightarrow$ Asn had significant influences on the elevation of MIC values $(P<0 \cdot 05)$.

Fluoroquinolones have been widely used to treat infections caused by $N$. gonorrhoeae since the 1980s because of their strong antibacterial activities, comparatively low costs and convenience of oral administration. However, resistance to these drugs emerged and spread quickly in the 1990s (Gu et al., 2004; Ye, 2001). Resistance to ciprofloxacin from $N$. gonorrhoeae isolated in Nanjing has increased from $2 \cdot 89 \%$ in 1994 to $97 \cdot 17 \%$ in 2002 (Su et al., 2004). Our results in this study indicated that the resistance rate of N. gonorrhoeae isolates from Nanjing, Xuzhou and Wuxi against ciprofloxacin has reached $100 \%$ (Table 1). It is indeed alarming to note such an increase in drug resistance of over 30 -fold within 10 years, and this antibiotic should not be recommended for treatment of gonococcal infections in China, especially in the Jiangsu Province. Other antibiotics, such as third- or fourth-generation cephalosporins or spectinomycin, should be considered.

It has been reported that earlier fluoroquinolone-resistant $N$. gonorrhoeae have mutations in the quinolone-resistant determining region (QRDR) (Belland et al., 1994). While susceptible bacteria have no mutation found in either $g y r A$ or parC (Shultz et al., 2001; Tanaka et al., 2000), when a single mutation in $g y r A$ is found in an isolate, there is an elevation of the fluoroquinolone MIC. In earlier studies by Belland et al. (1994) and Shultz et al. (2001), it has been demonstrated that resistance levels are correlated with sequential changes in $g y r A$ and parC mutations. Higher MICs were obtained when isolates had double gyrA mutations or co-existing parC mutations. Our present study revealed that nearly all the $N$. gonorrhoeae isolates were fluoroquinolone resistant (Table 1), and among the 54 isolates analysed for mutations, all demonstrated a change of Ser-91 $\rightarrow$ Phe in their gyrA gene, and all except one demonstrated a change in position 95 of the amino acid sequence (Table 2).

It was also demonstrated that most of the isolates with $g y r A$ mutations that had co-existing parC mutations were more resistant than those without parC mutations $(P<0 \cdot 05$; Table 3). Mutations in the parC gene were found to be mainly caused by amino acid changes in position 86 to 91, with 18 types (Table 2), excluding silent mutations. Previous studies of GyrA and ParC in N. gonorrhoeae have reported 12 amino acid changes in GyrA, including Ala-67 $\rightarrow$ Ser, Ala-75 $\rightarrow$ Ser, Ala- $84 \rightarrow$ Pro, His- $88 \rightarrow$ Gln, Ser91 $\rightarrow$ Phe, Ser-91 $\rightarrow$ Tyr, Ser-91 $\rightarrow$ Cys, Asp-95 $\rightarrow$ Gly, Asp95 $\rightarrow$ Ala, Asp-95 $\rightarrow$ Asn, Asn-103 $\rightarrow$ Ile and Val-120 $\rightarrow$ Leu; and 16 amino acid changes in ParC, including Gly-85 $\rightarrow$ Cys, Asp-86 $\rightarrow$ Asn, Ser-87 $\rightarrow$ Ile, Ser-87 $\rightarrow$ Asn, Ser- $87 \rightarrow$ Arg, Ser- $88 \rightarrow$ Arg, Ser- $88 \rightarrow$ Pro, Ser-88 $\rightarrow$ Thr, Glu-91 $\rightarrow$ Lys, Glu$91 \rightarrow$ Gly, Glu-91 $\rightarrow$ Gln, Ala-92 $\rightarrow$ Gly, Phe- $100 \rightarrow$ Leu, Phe$100 \rightarrow$ Tyr, Arg- $116 \rightarrow$ His and Arg-116 $\rightarrow$ Leu (Chaudhry et al., 2002; Shultz et al., 2001; Su \& Lind, 2001; Tanaka et al., 1998, 2000; Trees et al., 1999; Yoo et al., 2004). Our results concurred with most of the findings reported in the literature; in addition, we have found two new mutation sites of Ala-92 $\rightarrow$ Pro and Gln-102 $\rightarrow$ His in gyrA, and a new mutation of Ala-29 $\rightarrow$ Pro in parC (Table 2). We would speculate that there might be geographical differences among resistant isolates of $N$. gonorrhoeae (Shultz et al., 2001; Yong et al., 2004).

We further analysed the correlation between the number of mutation sites, their position, and the MIC results of the two fluoroquinolones. Through multi-regression analysis, it was revealed that the positions of mutations in the gyrA and parC genes significantly influenced resistance levels against fluoroquinolones, especially the amino acid changes Asp95 $\rightarrow$ Ala in GyrA, and Ser-87 $\rightarrow$ Arg and Ser-87 $\rightarrow$ Asn in ParC $(P<0 \cdot 05)$. Since the amino acid change Ser-91 $\rightarrow$ Phe in GyrA was found in all isolates, its effect on fluoroquinolones was not measurable through multi-regression analysis due to the lack of a control group. However, correlation of such a mutation to the MIC for fluoroquinolones has previously been demonstrated to be significant (Chaudhry et al., 2002; Shultz et al., 2001; Su \& Lind, 2001; Tanaka et al., 1998, 2000; Trees et al., 1999; Yoo et al., 2004). Although the detection of mutations in gyrB and parE was not performed in our study, an increase in the number of mutations would result in higher MICs for ciprofloxacin.

Our findings also revealed variations between mutations and levels of fluoroquinolone resistance in N. gonorrhoeae. Isolates with the same mutations demonstrated different MICs, and isolates with different mutations could give 
Table 2. Distribution of $g y r A$ and parC mutations in relation to fluoroquinolone MICs

\begin{tabular}{|c|c|c|c|c|c|c|c|c|c|c|}
\hline \multirow[t]{2}{*}{ gyrA mutation types (amino acid change) } & \multicolumn{7}{|c|}{ parC mutations with amino acid change } & \multirow{2}{*}{$\begin{array}{l}\text { Number of isolates } \\
\qquad(n=54)\end{array}$} & \multicolumn{2}{|c|}{ MIC range $\left(\mathrm{mg} \mathrm{l}^{-1}\right)$} \\
\hline & Ala-29 & Asp-86 & Ser-87 & Ser-87 & Ser-87 & Ser-88 & Glu-91 & & Ciprofloxacin & Levofloxacin \\
\hline \multirow[t]{4}{*}{ A $(n=19) \quad($ Ser-91 $\rightarrow$ Phe, Asp-95 $\rightarrow$ Gly $)$} & & & & & & & & 2 & $1-4$ & $1-4$ \\
\hline & & Asn & & & & & & 2 & $1-8$ & $2-8$ \\
\hline & & & & & & & Gly & 2 & $8-16$ & $4-8$ \\
\hline & & & Arg & & & & & 13 & $4-32$ & $2-8$ \\
\hline \multirow{8}{*}{ B $(n=24)($ Ser-91 $\rightarrow$ Phe, Asp-95 $\rightarrow$ Ala $)$} & & Asn & & & & & & 1 & 1 & 1 \\
\hline & & & & & & Pro & & 1 & 2 & 2 \\
\hline & & & & & & & Gly & 1 & 2 & 2 \\
\hline & Pro & & & Asn & & & & 1 & 2 & 2 \\
\hline & & & & & & & & 9 & $2-16^{*}$ & $2-8^{*}$ \\
\hline & & & & Asn & & & & 5 & $2-8$ & $1-8$ \\
\hline & & & Arg & & & & & 5 & $2-16$ & $1-8$ \\
\hline & & Asn & & & Ile & & & 1 & 8 & 4 \\
\hline \multirow[t]{4}{*}{ C $(n=9) \quad($ Ser-91 $\rightarrow$ Phe, Asp-95 $\rightarrow$ Asn $)$} & & & & & & & & 1 & 2 & 4 \\
\hline & & Asn & & & & & & 3 & $4-16$ & $4-8$ \\
\hline & & & $\operatorname{Arg}$ & & & & & 3 & $16-32$ & $8-16$ \\
\hline & & Asn & & & Ile & & & 2 & $32 \dagger$ & $16 \dagger$ \\
\hline $\mathrm{D}(n=1) \quad($ Ser-91 $\rightarrow$ Phe, Ala-92 $\rightarrow$ Pro $)$ & & & & & & & & 1 & 2 & 2 \\
\hline E $(n=1) \quad($ Ser-91 $\rightarrow$ Phe, Asp-95 $\rightarrow$ Asn, Gln-102 $\rightarrow$ His $)$ & & & $\operatorname{Arg}$ & & & & & 1 & 16 & 8 \\
\hline
\end{tabular}

${ }^{*}$ Only one isolate exhibited a MIC of $16 \mathrm{mg} \mathrm{l}^{-1}$ for ciprofloxacin and $8 \mathrm{mg} \mathrm{l}^{-1}$ for levofloxacin; the MIC range of the other eight isolates ranged from 2 to 4 mg $1^{-1}$

$\dagger$ The two isolates exhibited the same MIC values for ciprofloxacin and levofloxacin. 
Table 3. Number of parC mutations in relation to MICs for fluoroquinolones

\begin{tabular}{|c|c|c|c|c|c|c|}
\hline \multirow[t]{2}{*}{ Antibiotic } & \multirow{2}{*}{$\begin{array}{c}\text { Number of } \\
\text { parC mutations }\end{array}$} & \multirow{2}{*}{$\begin{array}{l}\text { Number of } \\
\text { isolates }\end{array}$} & \multirow{2}{*}{$\begin{array}{l}\text { Mean of } \\
\ln (\mathrm{MIC})\end{array}$} & \multirow[t]{2}{*}{ SD } & \multicolumn{2}{|c|}{ ANOVA analysis results } \\
\hline & & & & & F value & $P$ value \\
\hline \multirow[t]{3}{*}{ Ciprofloxacin } & 0 & 13 & $0 \cdot 96$ & $0 \cdot 76$ & $5 \cdot 67$ & $0 \cdot 006$ \\
\hline & 1 & 37 & $1 \cdot 93$ & $1 \cdot 02$ & & \\
\hline & 2 & 4 & $2 \cdot 43$ & $1 \cdot 33$ & & \\
\hline \multirow[t]{3}{*}{ Levofloxacin } & 0 & 13 & $1 \cdot 07$ & $0 \cdot 61$ & $1 \cdot 89$ & $0 \cdot 160$ \\
\hline & 1 & 37 & $1 \cdot 41$ & $0 \cdot 82$ & & \\
\hline & 2 & 4 & $1 \cdot 91$ & $1 \cdot 04$ & & \\
\hline
\end{tabular}

identical MIC results. It is highly likely that fluoroquinolone resistance involves mechanism(s) other than mutations in the gyrA and/or parC genes, as found with other bacterial species, which would require further investigation.

\section{REFERENCES}

Belland, R. J., Morrison, S. G., Ison, C. \& Huang, W. M. (1994). Neisseria gonorrhoeae acquires mutations in analogous regions of gyrA and parC in fluoroquinolone-resistant isolates. Mol Microbiol 14, 371-380.

Cai, C. J., Wu, C. H., Lu, S. C., Zhen, H. P., Chen, S. W. \& Liang, L. H. (2003). Study on antibiotic resistance in Neisseria gonorrhoeae in Zhongshan city. Guangdong Medical J 24, 1123-1124.

Centers for Disease Control and Prevention (2005). Neisseria Gonorrhoeae reference strains for antimicrobial susceptibility testing, 10 pp. http://www.cdc.gov/std/Gonorrhea/arg/B88-Feb-2005.pdf. Atlanta, GA: Centers for Disease Control and Prevention.

Chaudhry, U., Ray, K., Bala, M. \& Saluja, D. (2002). Mutation patterns in gyrA and parC genes of ciprofloxacin resistant isolates of Neisseria gonorrhoeae from India. Sex Transm Infect 78, 440-444.

Chinese Centre for Disease Control and Prevention (2004). Chinese Centre for Disease Control and Prevention. Centre for Public Health Surveillance and Information Service. National data on class A and B infectious diseases in December, 2003. Disease Surveillance (Beijing) 19, 3.

Gu, W. M., Yang, Y., Wu, L. \& 7 other authors (2004). Surveillance on antibiotic susceptibility of Neisseria Gonorrhoeae from 1988 to 2002 in Shanghai. Chin J Dermatol 37, 323-325.

Hawkey, P. M. (2003). Mechanism of quinolone action and microbial response. J Antimicrob Chemother 51 (Suppl. S1), 29-35.

Krishna, R., Manju, B., Sudarshan, K. \& Jai, P. N. (2005). Antimicrobial resistance of Neisseria gonorrhoeae in selected World Health Organization Southeast Asia Region countries: an overview. Sex Transm Dis 32, 178-184.

National Committee for Clinical Laboratory Standards (2002). Performance Standard for Antimicrobial Susceptibility Testing: Twelfth Informational Supplement M100-S12. Wayne, PA: National Committee for Clinical Laboratory Standards.

Ng, L. K., Sawatzky, P., Martin, I. E. \& Booth, S. (2002). Characterization of ciprofloxacin resistance in Neisseria gonorrhoeae isolates in Canada. Sex Transm Dis 29, 780-788.

Shigemura, K., Shirakawa, T., Okada, H. \& 8 other authors (2004). Mutations in the $g y r A$ and parC genes and in vitro activities of fluoroquinolones in 91 clinical isolates of Neisseria gonorrhoeae in Japan. Sex Transm Dis 31, 180-184.
Shultz, T. R., Tapsall, J. W. \& White, P. A. (2001). Correlation of in vitro susceptibilities to newer quinolones of naturally occurring quinolone-resistant Neisseria gonorrhoeae strains with changes in GyrA and ParC. Antimicrob Agents Chemother 45, 734-738.

Su, X. H. (2002). Drug resistance of gonococcus and treatment of gonorrhoeae. J Clin Dermatol 31, 601-603.

Su, X. \& Lind, I. (2001). Molecular basis of high-level ciprofloxacin resistance in Neisseria gonorrhoeae strains isolated in Denmark from 1995 to 1998. Antimicrob Agents Chemother 45, 117-123.

Su, X. H., Dai, X. Q., Sun, H. H. \& Ye, S. Z. (2004). Surveillance of antimicrobial susceptibilities of Neisseria gonorrhoeae in Nanjing City, 1999-2002. Chin J Dermatol 37, 262-264.

Tanaka, M., Takahashi, K., Saika, T., Kobayashi, I., Ueno, T. \& Kumazawa, J. (1998). Development of fluoroquinolone resistance and mutations involving GyrA and ParC proteins among Neisseria gonorrhoeae isolates in Japan. J Urol 159, 2215-2219.

Tanaka, M., Nakayama, H., Haraoka, M. \& Saika, T. (2000). Antimicrobial resistance of Neisseria gonorrhoeae and high prevalence of ciprofloxacin-resistant isolates in Japan, 1993-1998. J Clin Microbiol 38, 521-525.

Tanaka, M., Nakayama, H., Notomi, T., Irie, S., Tsunoda, Y., Okadome, A., Saika, T. \& Kobayashi, I. (2004). Antimicrobial resistance of Neisseria gonorrhoeae in Japan, 1993-2002: continuous increasing of ciprofloxacin-resistant isolates. Int J Antimicrob Agents 24 (Suppl. 1), S15-S22.

Trees, D. L., Sandul, A. L., Peto-Mesola, V., Aplasca, M. R., Leng, H. B., Whittington, W. L. \& Knapp, J. S. (1999). Alterations within the quinolone resistance-determining regions of GyrA and ParC of Neisseria gonorrhoeae isolated in the Far East and the United States. Int J Antimicrob Agents 12, 325-332.

World Health Organization (2003). Surveillance of antibiotic resistance in Neisseria gonorrhoeae in the WHO Western Pacific Region, 2002. Commun Dis Intell 27, 488-491.

Ye, S. Z. (2001). Surveillance of antibiotic susceptibility of Neisseria gonorrhoeae isolated from ten cities of China from 1993-1998. Chin J Dermatol 34, 175-177.

Yong, D., Kim, T. S., Choi, J. R., Yum, J. H., Lee, K., Chong, Y., Oh, H. B., Shultz, T. \& Tapsall, J. W. (2004). Epidemiological characteristics and molecular basis of fluoroquinolone-resistant Neiserria gonorrhoeae strains isolated in Korea and nearby countries. J Antimicrob Chemother 54, 451-455.

Yoo, J., Yoo, C., Cho, Y., Park, H., Oh, H. B. \& Seong, W. K. (2004). Antimicrobial resistance patterns (1999-2002) and characterization of ciprofloxacin-resistant Neisseria gonorrhoeae in Korea. Sex Transm Dis 31, 305-310.

Zhu, B. Y. \& Zhao, X. M. (2003). The analysis of antibiotic resistance in Neisseria gonorrhoeae. J Clin Dermatol 32, 199-200. 